EPJ Web of Conferences 81, 05002 (2014)

DOI: $10.1051 /$ epjconf/ 20148105002

C Owned by the authors, published by EDP Sciences, 2014

\title{
Low energy structure of the nucleon from chiral effective field theory
}

\author{
Jose Manuel Alarcón ${ }^{1, a}$ \\ ${ }^{1}$ Cluster of Excellence PRISMA Institut für Kernphysik, \\ Johannes Gutenberg-Universität, Mainz D-55099, Germany
}

\begin{abstract}
We present some updated results regarding the scalar and electromagnetic structure of the nucleon obtained by the relativistic formulation of chiral effective field theory with baryons. We compare them with previous determinations available in the literature, and show their relevance for searches of physics beyond the standard model in the low energy frontier.
\end{abstract}

\section{Introduction}

An accurate knowledge of the structure of the nucleon is important to improve our understanding of the fundamental interactions and provide theoretical estimations of some important quantities with a small uncertainty. This is specially relevant for experiential searches of physics beyond the standard model in the low energy frontier. The electromagnetic structure of the nucleon is a good example, since its impact in the muonic hydrogen Lamb shift has the potential to solve the so-called "proton radius puzzle". In fact, this has motivated many theoretical works [1-10]. Also, a good understanding of the scalar structure of the nucleon is required to answer many important questions, as the origin of the mass of the ordinary matter, or to learn how different fundamental elements needed for life appear in nature [11]. Nowadays, an accurate knowledge of the scalar structure of the nucleon is also important in dark matter (DM) detection, since the different results regarding its scalar coupling is the main hadronic uncertainty in the estimation of the spin-independent DMnucleon elastic scattering cross sections [12-14].

These properties mentioned above are governed by the strong interaction, described by the quantum chromo dynamics (QCD). Unfortunately, QCD does not allow a perturbative treatment in the low energy region, which is the region of interest here. Fortunately, one can always construct an effective field theory (EFT) containing the symmetries of QCD and its relevant degrees of freedom to apply perturbative techniques. This approach exists since long time ago and is called chiral effective field theory.

In this contribution I will present some recent results concerning the study of the scalar and electromagnetic structure of the nucleon in the relativistic formulation of chiral EFT with baryons. The main difference with respect to the other versions of chiral EFT with baryons (i. e. Heavy Baryon (HB) [15] and Infrared Regularization

\footnotetext{
ae-mail: alarcon@kph.uni-mainz.de
}

[16]) is that this is the only one that preserves the relativistic structure of the original formulation of Gasser, Sainio and Svarc [17], while keeping the correct analytical strucure $[18,19]$. This is achieved thanks to the Extended-OnMass-Shell (EOMS) renormalization scheme [22]. The application of this formalism has given improved results in the $\pi N$ sector [19-21], as well as in double virtual Compton scattering $[8,23,24]$.

\section{Scalar Structure of the Nucleon}

From the theory side, the scalar structure of the nucleon is crucial to understand the origin of the mass of the ordinary matter. In what concerns to experimental searches of physics beyond the Standard Model, the scalar form factor of the nucleon at zero momentum transfer is a required quantity for the estimation of dark matter detection. In fact, the pion-nucleon sigma term $\left(\sigma_{\pi N}\right)$ and the strange sigma term $\left(\sigma_{s}\right)$, defined as

$$
\begin{aligned}
\sigma_{\pi N} & =\frac{\hat{m}}{2 m_{N}}\langle N|(\bar{u} u+\bar{d} d)| N\rangle \\
\sigma_{s} & =\frac{m_{s}}{2 m_{N}}\langle N|\bar{s} s| N\rangle
\end{aligned}
$$

are the main hadronic uncertainties in the estimation of dark matter-nucleon spin independent elastic scattering cross sections. Where, in the previous equations, $m_{N}$ is the nucleon mass, $\hat{m}=\left(m_{u}+m_{d}\right) / 2$ and $m_{u}, m_{d}$ and $m_{s}$ are the masses of the up, down and strange quarks.

The traditional way to access to $\sigma_{\pi N}$ was through the Cheng-Dashen theorem [25]. It connects the value of the Born-subtracted isoscalar scattering amplitude, $\bar{D}^{+}(v, t)$, to the value of the scalar form factor of the nucleon at $t=2 M_{\pi}^{2}, \sigma\left(t=2 M_{\pi}^{2}\right)$. On the other hand, $\sigma\left(t=2 M_{\pi}^{2}\right)$ can be connected to the sigma-term $\sigma(t=0)=\sigma_{\pi N}$ via dispersion relations. The Cheng-Dashen theorem then allow us to write [17]: 


$$
\begin{aligned}
\Sigma \equiv f_{\pi}^{2} \bar{D}^{+}\left(0,2 M_{\pi}^{2}\right)= & \sigma\left(t=2 M_{\pi}^{2}\right)+\Delta_{R} \\
& =\sigma_{\pi N}+\Delta_{\sigma}+\Delta_{R},
\end{aligned}
$$

where $\Delta_{\sigma}$ and $\Delta_{R}$ are two known quantities [26, 27], $M_{\pi}$ is the mass of the pion, $v=(s-u) / 4 m_{N}$, and $s, t$ and $u$ are the Mandelstam variables [19]. Through dispersive methods one can provide an analytic representation of this amplitude satisfying unitarity and causality, which can be extrapolated to the Cheng-Dashen point $\left(v=0, t=2 M_{\pi}^{2}\right)$ to extract the value of $\sigma_{\pi N}$. However, since different dispersive analyses give incompatible results [28], it was not clear whether this was due to the different experimental information used to reconstruct the scattering amplitude, or was due to an underestimation of systematic error in the extrapolation to the Cheng-Dashen point.

The advantage of the chiral EFT is that one can calculate directly this quantity in a perturbative framework in which one has better control of the systematic uncertainties. However, the main disadvantage is that the chiral expansion of the sigma-term is governed by a low-energy constant (LEC). In its relativistic form, the sigma-term has, up to order $O\left(p^{3}\right)$, the following form

$$
\begin{aligned}
\sigma_{\pi N} & =-4 c_{1} M_{\pi}^{2}-\frac{3 g_{A}^{2} M_{\pi}^{3}}{16 \pi^{2} f_{\pi}^{2} m_{N}} \\
& \times\left(\frac{3 m_{N}^{2}-M_{\pi}^{2}}{\sqrt{4 m_{N}^{2}-M_{\pi}^{2}}} \arccos \frac{M_{\pi}}{2 m_{N}}+M_{\pi} \log \frac{M_{\pi}}{m_{N}}\right),
\end{aligned}
$$

where one sees clearly that the leading order contribution comes from $c_{1}$. This LEC can be extracted by fitting the chiral calculation of the $\pi N$ scattering amplitude to the existing data. However, this extraction is not so trivial due to the problems of convergence of the perturbative calculation, which gives rise to unnatural values of the LECs [19]. The reason of this lack of convergence can be traced back to the importance of the $\Delta(1232)$ in $\pi N$ scattering, since it is very close to the threshold of the reaction and couples strongly to $\pi N$. One can overcome this problem by including the $\Delta$ as an explicit degree of freedom in the EFT formulation. This inclusion, together with the correct analytic structure of the amplitude given by the relativistic formulation, was crucial to achieve an improved description of the $\pi N$ scattering phenomenology [19]. This improvement allowed us to extract, from the partial wave analyses (PWAs) data, the value of $\sigma_{\pi N}$ [29]. In this work we show that the different results of the dispersive analyses are due to the data employed, and give evidences that favours the modern data as source of extraction of the sigma-term. Based on this modern data, we obtain a value of [29]

$$
\sigma_{\pi N}=59(7) \mathrm{MeV} .
$$

The same approach was used in the $S U(3)_{F}$ sector to investigate the strangeness content of the nucleon [30]. This information can be obtained through (2). According to the Hellmann-Feynmann theorem, this equation can be written as the derivative of the nucleon mass with respect to the mass of the strange quark

$$
\sigma_{s}=m_{s} \frac{\partial}{\partial m_{s}} m_{N} .
$$

In fact, Eq. (6) is very convenient for the chiral approaches, since chiral EFT allows us to calculate the dependence of the nucleon mass on the masses of the quarks. As in the $S U(2)_{F}$ sector, this dependence is given in terms of unknown LECs. However, up to the order of our calculation, they can be determined from the physical values of the baryon octet masses and $\sigma_{\pi N}$. In Ref. [30] we updated the previous results with the relativistic approach including explicitly the decuplet contribution and obtained

$$
\sigma_{s}=16(80)(60) \mathrm{MeV}
$$

where the errors are from statistical and systematic origin, respectively.

\section{Electromagnetic Structure of the Nucleon}

Polarizability is a property of any composite charged system. They give information about how the system, in this case the nucleon, reacts under electromagnetic probes. They have become more interesting lately due to their impact on the theoretical estimation of the muonic hydrogen Lamb shift, because it can solve the so-called "proton radius puzzle". The origin of this puzzle is the discrepancy of the proton charge radius extracted from electronic measurements [31] (as $e^{-} p$ scattering and normal hydrogen Lamb shift) and the $\mu H$ Lamb shift $[32,33]$. However, it has been recently claimed that the extrapolation of the electric form factor used to extract the radius could be the source of the discrepancy between the $e^{-} p$ scattering and $\mu H$ Lamb shift extractions $[34,35]$. The importance of the polarizabilities in this puzzle is that, since the muon in the $\mu \mathrm{H}$ atom is much closer to the proton, it is much more sensitive to its internal electromagnetic structure, what can affect considerably the extraction of the radius in terms of the Lamb shift. The interest in the chiral calculation of this contribution is that one can provide a prediction for the leading contribution on QCD grounds. Also, it is important to realize that the main contribution of the two photon exchange corrections, where the polarizabilities enter, comes from the region of low $Q^{2}$, see Eq. (9). This is a region of energies where chiral EFT is a reliable approach. In Fig. 1, one can see the kind of contributions generated by the polarizabilities, that would come from the inelastic (non-Born) part of the Compton tensor $T^{\mu \nu}$ :

$$
\begin{aligned}
T^{\mu \nu} & =\left(-g^{\mu \nu}+\frac{q^{\mu} q^{v}}{q^{2}}\right) T_{1}\left(v, Q^{2}\right) \\
& +\frac{1}{m_{N}^{2}}\left(P^{\mu}-\frac{P \cdot q}{q^{2}} q^{\mu}\right)\left(P^{v}-\frac{P \cdot q}{q^{2}} q^{v}\right) T_{2}\left(v, Q^{2}\right) .
\end{aligned}
$$

As was shown in Ref. [8], the correction to the Lamb shift due to the polarizabilities $\left(\Delta E^{(p o l)}\right)$ can be well approximated by 


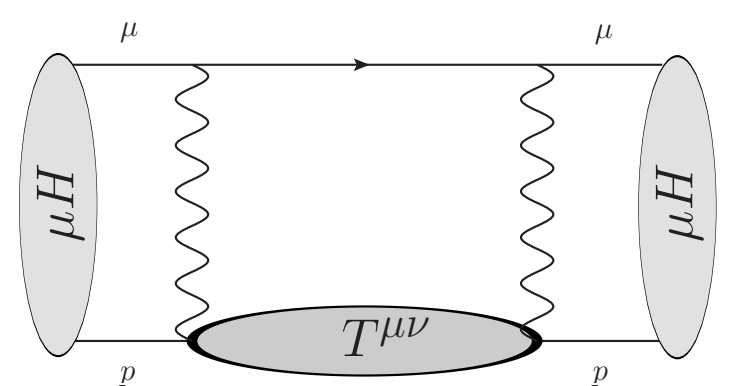

Figure 1. Two-photon exchange correction to the Coulomb potential, direct diagram.

$$
\begin{aligned}
\Delta E_{2 S}^{(p o l)} \approx \frac{\alpha_{e m}}{\pi} \phi_{n=}^{2} & \int_{0}^{\infty} \frac{d Q}{Q^{2}}\left[\sqrt{1+\frac{Q^{2}}{4 m_{\ell}^{2}}}-\frac{Q}{2 m_{\ell}}\right] \\
& \times\left[T_{1}^{(N B)}\left(0, Q^{2}\right)-T_{2}^{(N B)}\left(0, Q^{2}\right)\right]
\end{aligned}
$$

where $m_{\ell}$ is the mass of the lepton, $\alpha_{e m}$ is the fine structure constant, $\phi_{n}$ is the hydrogen wave function at the origin, and $T_{1}^{(N B)}$ and $T_{2}^{(N B)}$ are the non-Born parts of $T_{1}$ and $T_{2}$.

In Ref. [8] we showed the prediction of relativistic chiral EFT compared to the HB results and phenomenological determinations. A summary of these is shown in Table 1, where the relativistic result is labeled as LO-BChPT. One observes a big difference between the $\mathrm{HB}$ and the relativistic calculations, the second one being much closer to the phenomenological determinations based on experimental information. Investigating the difference between the two chiral EFT approaches one sees a much faster convergence in the relativistic formulation than in the $\mathrm{HB}$ one. This is illustrated in Fig. 2, where we show the result for $\Delta E^{(p o l)}$ depending on the maximum $Q^{2}$ up to which one integrates, $Q_{\text {max }}^{2}$. The numerical values on the right of the frame correspond to the asymptotic result $\left(Q_{\max }^{2} \rightarrow \infty\right)$. One sees that, while the relativistic result converges in the region of low $Q^{2}$, the HB result differs from its asymptotic result more than the systematic error of the calculation. Therefore one can conclude that once one keeps the relativistic structure of the Compton amplitude, the prediction for $\Delta E^{(p o l)}$ converges much faster and gives a result very close to the phenomenological determinations. This is a remarkable result since the chiral calculation presented here is a prediction based on the low-energy symmetries of QCD, while the phenomenological determinations depend mostly on experimental information.

\section{Conclusions}

The properties of the nucleons at low energies are relevant for experimental searches of physics beyond the standard model. They can be accessed through an effective field theory, like chiral EFT, containing the symmetries of QCD relevant for the energies of interest. Thanks to the recent developments in the formulation of chiral EFT with baryons one can have a deeper insight into the scalar

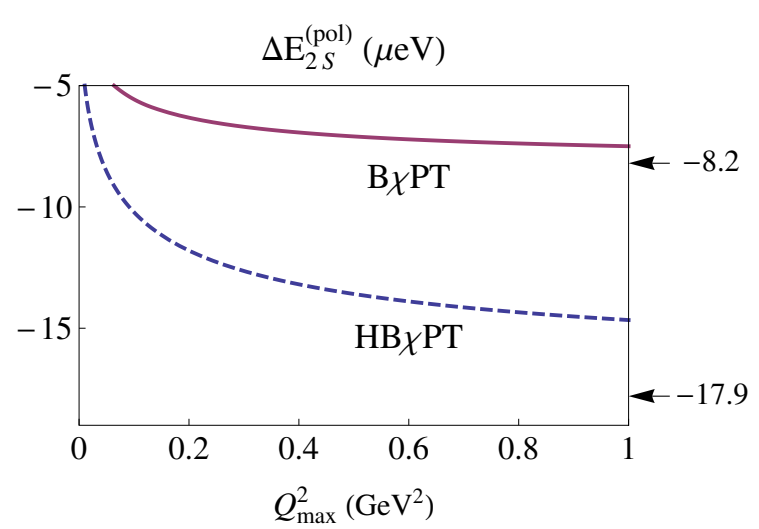

Figure 2. Dependence of the integral (9) on the region of integration.

and electromagnetic structure of the nucleon. An accurate knowledge of these is relevant for dark matter searches and the proton radius puzzle. In this regard, we showed that the relativistic EFT formulation with baryons, in combination with modern data, points to a relatively large value of the pion-nucleon sigma term, while keeping a small strangeness content of the nucleon. On the other hand, we also showed that the chiral EFT prediction of the polarizabilities contribution to the $\mu H$ Lamb shift is in excellent agreement with the latest phenomenological determinations, improving the previous EFT predictions. These two examples show the potential of chiral EFT to provide information useful in experimental searches of physics beyond the standard model in the low-energy frontier.

\section{Acknowledgements}

These works were partially supported by the Deutsche Forschungsgemeinschaft (DFG) through the Collaborative Research Center "The Low-Energy Frontier of the Standard Model" (SFB 1044), by the Cluster of Excellence "Precision Physics, Fundamental Interactions and Structure of Matter" (PRISMA).

\section{References}

[1] K. Pachucki, Phys. Rev. A 60, 3593 (1999).

[2] A. P. Martynenko, Phys. Atom. Nucl. 69, 1309 (2006).

[3] D. Nevado and A. Pineda, Phys. Rev. C 77, 035202 (2008).

[4] C. E. Carlson and M. Vanderhaeghen, Phys. Rev. A 84, 020102 (2011).

[5] M. C. Birse and J. A. McGovern, Eur. Phys. J. A 48, 120 (2012).

[6] M. Gorchtein, F. J. Llanes-Estrada and A. P. Szczepaniak, Phys. Rev. A 87, 052501 (2013).

[7] R. J. Hill and G. Paz, Phys. Rev. Lett. 107, 160402 (2011).

[8] J. M. Alarcon, V. Lensky and V. Pascalutsa, Eur. Phys. J. C 74, 2852 (2014).

[9] C. Peset and A. Pineda, arXiv:1403.3408 [hep-ph]. 


\begin{tabular}{ccccccccc}
\hline & & Marty- & Nevado \& & Carlson \& & Birse \& & Gorchtein & & Peset \& \\
& Pachucki & nenko & Pineda & Vanderhaeghen & McGovern & et al. & LO-BChPT & Pineda \\
$(\mu \mathrm{eV})$ & {$[1]$} & {$[2]$} & {$[3]$} & {$[4]$} & {$[5]$} & {$[6]$} & {$[8]$} & {$[9,10]$} \\
\hline$\Delta E_{2 S}^{(\mathrm{pol})}$ & $-12(2)$ & -11.5 & -18.5 & $-7.4(2.4)$ & $-8.5(1.1)$ & $-15.3(5.6)$ & $-8.2\left({ }_{-2.5}^{+1.2}\right)$ & -26.5 \\
\hline
\end{tabular}

Table 1. Summary of available calculations of the polarizability effects on the $2 S$ level of $\mu \mathrm{H}$.

[10] C. Peset and A. Pineda, arXiv:1406.4524 [hep-ph].

[11] J. C. Berengut, E. Epelbaum, V. V. Flambaum, C. Hanhart, U. -G. Meissner, J. Nebreda and J. R. Pelaez, Phys. Rev. D 87, no. 8, 085018 (2013).

[12] A. Bottino, F. Donato, N. Fornengo and S. Scopel, Astropart. Phys. 13, 215 (2000).

[13] A. Bottino, F. Donato, N. Fornengo and S. Scopel, Astropart. Phys. 18, 205 (2002).

[14] J. R. Ellis, K. A. Olive and C. Savage, Phys. Rev. D 77, 065026 (2008).

[15] E. E. Jenkins and A. V. Manohar, Phys. Lett. B 255 558 (1991).

[16] T. Becher and H. Leutwyler, Eur. Phys. J. C 9, 643 (1999).

[17] J. Gasser, M. E. Sainio and A. Svarc, Nucl. Phys. B 307, 779 (1988).

[18] J. M. Alarcon, J. M. Camalich, J. A. Oller and L. Alvarez-Ruso, Phys. Rev. C 83055205 (2011).

[19] J. M. Alarcon, J. Martin Camalich and J. A. Oller, Annals Phys. 336, 413 (2013).

[20] Y. -H. Chen, D. -L. Yao and H. Q. Zheng, Phys. Rev. D 87, no. 5, 054019 (2013).

[21] D. Siemens, V. Bernard, E. Epelbaum, H. Krebs and U.-G. Meißner, arXiv:1403.2510 [nucl-th].

[22] T. Fuchs, J. Gegelia, G. Japaridze and S. Scherer, Phys. Rev. D 68, 056005 (2003).
[23] V. Bernard, E. Epelbaum, H. Krebs and U.G. Meißner, Phys. Rev. D 87, 054032 (2013).

[24] V. Lensky, J. M. Alarcón and V. Pascalutsa, arXiv:1407.2574 [hep-ph].

[25] T. P. Cheng, R. F. Dashen, Phys. Rev. Lett. 26594 (1971) .

[26] V. Bernard, N. Kaiser and U. -G. Meissner, Phys. Lett. B 389, 144 (1996).

[27] M. Hoferichter, C. Ditsche, B. Kubis and U. G. Meissner, JHEP 1206, 063 (2012).

[28] M. M. Pavan, I. I. Strakovsky, R. L. Workman and R. A. Arndt, PiN Newslett. 16, 110 (2002).

[29] J. M. Alarcón, J. Martin Camalich and J. A. Oller, Phys. Rev. D 85, 051503 (2012).

[30] J. M. Alarcon, L. S. Geng, J. Martin Camalich and J. A. Oller, Phys. Lett. B 730, 342 (2014).

[31] J. C. Bernauer et al., Phys. Rev. Lett. 105, 242001 (2010).

[32] R. Pohl, et al., Nature 466, 213 (2010).

[33] A. Antognini, et al., Science 339, 417 (2013).

[34] I. T. Lorenz, H. -W. Hammer and U.-G. Meißner, Eur. Phys. J. A 48, 151 (2012).

[35] I. T. Lorenz and U.-G. Meißner, Phys. Lett. B (2014), pp. 57-59. 